\title{
Robust Stability and Control of Linear Interval Parameter Systems Using Quantitative (State Space) and Qualitative (Ecological) Perspectives
}

\author{
Rama K. Yedavalli and Nagini Devarakonda \\ The Ohio State University \\ United States of America
}

\section{Introduction}

The problem of maintaining the stability of a nominally stable linear time invariant system subject to linear perturbation has been an active topic of research for quite some time. The recent published literature on this 'robust stability' problem can be viewed mainly from two perspectives, namely i) transfer function (input/output) viewpoint and ii) state space viewpoint. In the transfer function approach, the analysis and synthesis is essentially carried out in frequency domain, whereas in the state space approach it is basically carried out in time domain. Another perspective that is especially germane to this viewpoint is that the frequency domain treatment involves the extensive use of 'polynomial' theory while that of time domain involves the use of 'matrix' theory. Recent advances in this field are surveyed in [1]-[2].

Even though in typical control problems, these two theories are intimately related and qualitatively similar, it is also important to keep in mind that there are noteworthy differences between these two approaches ('polynomial' vs 'matrix') and this chapter (both in parts I and II) highlights the use of the direct matrix approach in the solution to the robust stability and control design problems.

\section{Uncertainty characterization and robustness}

It was shown in [3] that modeling errors can be broadly categorized as i) parameter variations, ii) unmodeled dynamics iii) neglected nonlinearities and finally iv) external disturbances. Characterization of these modeling errors in turn depends on the representation of dynamic system, namely whether it is a frequency domain, transfer function framework or time domain state space framework. In fact, some of these can be better captured in one framework than in another. For example, it can be argued convincingly that real parameter variations are better captured in time domain state space framework than in frequency domain transfer function framework. Similarly, it is intuitively clear that unmodeled dynamics errors can be better captured in the transfer function framework. By similar lines of thought, it can be safely agreed that while neglected nonlinearities can be better captured in state space framework, neglected disturbances can 
be captured with equal ease in both frameworks. Thus it is not surprising that most of the robustness studies of uncertain dynamical systems with real parameter variations are being carried out in time domain state space framework and hence in this chapter, we emphasize the aspect of robust stabilization and control of linear dynamical systems with real parameter uncertainty.

Stability and performance are two fundamental characteristics of any feedback control system. Accordingly, stability robustness and performance robustness are two desirable (sometimes necessary) features of a robust control system. Since stability robustness is a prerequisite for performance robustness, it is natural to address the issue of stability robustness first and then the issue of performance robustness.

Since stability tests are different for time varying systems and time invariant systems, it is important to pay special attention to the nature of perturbations, namely time varying perturbations versus time invariant perturbations, where it is assumed that the nominal system is a linear time invariant system. Typically, stability of linear time varying systems is assessed using Lyapunov stability theory using the concept of quadratic stability whereas that of a linear time invariant system is determined by the Hurwitz stability, i.e. by the negative real part eigenvalue criterion. This distinction about the nature of perturbation profoundly affects the methodologies used for stability robustness analysis.

Let us consider the following linear, homogeneous, time invariant asymptotically stable system in state space form subject to a linear perturbation $E$ :

$$
\dot{x}=\left(A_{0}+E\right) x \quad x(0)=x_{0}
$$

where $A_{0}$ is an $n \times n$ asymptotically stable matrix and $E$ is the error (or perturbation) matrix. The two aspects of characterization of the perturbation matrix $E$ which have significant influence on the scope and methodology of any proposed analysis and design scheme are i) the temporal nature and ii) the boundedness nature of $E$. Specifically, we can have the following scenario:

i. Temporal Nature:

$$
\begin{array}{r}
\text { Time invariant error } \\
E=\text { constant }
\end{array} \quad \text { vs } \quad \begin{aligned}
& \text { Time varying error } \\
& E=E(t)
\end{aligned}
$$

ii. Boundedness Nature:

$$
\begin{array}{r}
\text { Unstructured } \\
\text { (Norm bounded) }
\end{array} \quad \text { vs } \quad \begin{aligned}
& \text { Structured } \\
& \text { (Elemental bounds) }
\end{aligned}
$$

The stability robustness problem for linear time invariant systems in the presence of linear time invariant perturbations (i.e. robust Hurwitz invariance problem) is basically addressed by testing for the negativity of the real parts of the eigenvalues (either in frequency domain or in time domain treatments), whereas the time varying perturbation case is known to be best handled by the time domain Lyapunov stability analysis. The robust Hurwitz invariance problem has been widely discussed in the literature essentially using the polynomial approach [4]-[5]. In this section, we address the time varying perturbation case, mainly motivated by the fact that any methodology which treats the time varying case can always be specialized to the time invariant case but not vice versa. However, we pay a price for the same, namely conservatism associated with the results when applied to the time invariant perturbation case. A methodology specifically tailored to time invariant perturbations is discussed and included by the author in a separate publication [6]. 
It is also appropriate to discuss, at this point, the characterization with regard to the boundedness of the perturbation. In the so called 'unstructured' perturbation, it is assumed that one cannot clearly identify the location of the perturbation within the nominal matrix and thus one has simply a bound on the norm of the perturbation matrix. In the 'structured' perturbation, one has information about the location(s) of the perturbation and thus one can think of having bounds on the individual elements of the perturbation matrix. This approach can be labeled as 'Elemental Perturbation Bound Analysis (EPBA)'. Whether 'unstructured' norm bounded perturbation or 'structured' elemental perturbation is appropriate to consider depends very much on the application at hand. However, it can be safely argued that 'structured' real parameter perturbation situation has extensive applications in many engineering disciplines as the elements of the matrices of a linear state space description contain parameters of interest in the evolution of the state variables and it is natural to look for bounds on these real parameters that can maintain the stability of the state space system.

\section{Robust stability and control of linear interval parameter systems under state space framework}

In this section, we first give a brief account of the robust stability analysis techniques in 3.1 and then in subsection 3.2 we discuss the robust control design aspect.

\subsection{Robust stability analysis}

The starting point for the problem at hand is to consider a linear state space system described by

$$
\dot{x}(t)=\left[A_{0}+E\right] x(t)
$$

where $x$ is an $\mathrm{n}$ dimensional state vector, asymptotically stable matrix and $E$ is the 'perturbation' matrix. The issue of 'stability robustness measures' involves the determination of bounds on $E$ which guarantee the preservation of stability of (1). Evidently, the characterization of the perturbation matrix $E$ has considerable influence on the derived result. In what follows, we summarize a few of the available results, based on the characterization of $E$.

1. Time varying (real) unstructured perturbation with spectral norm: Sufficient bound For this case, the perturbation matrix $E$ is allowed to be time varying, i.e. $E(t)$ and a bound on the spectral norm $\left(\sigma_{\max }(E(t))\right.$ where $\sigma(\cdot)$ is the singular value of $\left.(\cdot)\right)$ is derived. When a bound on the norm of $E$ is given, we refer to it as 'unstructured' perturbation. This norm produces a spherical region in parameter space. The following result is available for this case [7]-[8]:

$$
\sigma_{\max }(E(t))<\frac{1}{\sigma_{\max }(P)}
$$

where $P$ is the solution to the Lyapunov matrix

$$
P A_{0}+A_{0}^{T} P+2 I=0
$$

See Refs [9],[10],[11] for results related to this case. 


\section{Time varying (real) structured variation}

Case 1: Independent variations (sufficient bound) [12]-[13]

$$
\begin{gathered}
E_{i j}(t) \leq \forall_{t}\left|E_{i j}(t)\right|_{\max }=\varepsilon_{i j} \\
\varepsilon=\operatorname{Max}_{i j} \varepsilon_{i j} \\
\varepsilon_{i j}<\frac{1}{\sigma_{\max }\left(P_{m} U_{e}\right)_{s}} U_{e i j}
\end{gathered}
$$

where $P$ satisfies equation (3) and $U_{o i j}=\varepsilon_{i j} / \varepsilon$. For cases when $\varepsilon_{i j}$ are not known, one can take $U_{e i j}=\left|A_{o i j}\right| /\left|A_{o i j}\right|_{\max } \cdot(\cdot)_{\mathrm{m}}$ denotes the matrix with all modulus elements and $(\cdot)_{\mathrm{s}}$ denotes the symmetric part of $(\cdot)$.

3. Time invariant, (real) structured perturbation $E_{i j}=$ Constant

Case i: Independent Variations [13]-[15]: (Sufficient Bounds). For this case, E can be characterized as

$$
E=S_{1} D S_{2}
$$

where $S_{1}$ and $S_{2}$ are constant, known matrices and $\left|D_{i j}\right| \leq d_{i j} d$ with $d_{i j} \geq 0$ are given and $d>0$ is the unknown. Let $U$ be the matrix elements $U_{i j}=d_{i j}$. Then the bound on $d$ is given by [13]

$$
d<\frac{1}{\operatorname{Sup}_{\omega>0}\left(\left[S_{2}\left(j \omega I-A_{0}\right)^{-1} S_{1}\right]_{m} U\right)}=\mu_{J}=\mu_{Q}
$$

Notice that the characterization of $E$ (with time invariant) in (4) is accommodated by the characterization in [15]. $\rho(\cdot)$ is the spectral radius of $(\cdot)$.

Case ii: Linear Dependent Variation: For this case, $E$ is characterized (as in (6) before), by

$$
E=\sum_{i=1}^{r} \beta_{i} E_{i}
$$

and bounds on $\left|\beta_{i}\right|$ are sought. Improved bounds on $\left|\beta_{i}\right|$ are presented in [6].

This type of representation represents a 'polytope of matrices' as discussed in [4]. In this notation, the interval matrix case (i.e. the independent variation case) is a special case of the above representation where $E i$ contains a single nonzero element, at a different place in the matrix for different $i$.

For the time invariant, real structured perturbation case, there are no computationally tractable necessary and sufficient bounds either for polytope of matrices or for interval matrices (even for a $2 \times 2$ case). Even though some derivable necessary and sufficient conditions are presented in [16] for any general variation in $E$ (not necessarily linear dependent and independent case), there are no easily computable methods available to determine the necessary and sufficient bounds at this stage of research. So most of the research, at this point of time, seems to aim at getting better (less conservative) sufficient bounds. The following example compares the sufficient bounds given in [13]-[15] for the linear dependent variation case. 
Let us consider the example given in [15] in which the perturbed system matrix is given by

$$
\left(A_{0}+B K C\right)=\left[\begin{array}{ccc}
-2+k_{1} & 0 & -1+k_{1} \\
0 & -3+k_{2} & 0 \\
-1+k_{1} & -1+k_{2} & -4+k_{1}
\end{array}\right]
$$

Taking the nominally stable matrix to be

$$
A_{0}=\left[\begin{array}{ccc}
-2 & 0 & -1 \\
0 & -3 & 0 \\
-1 & -1 & -4
\end{array}\right]
$$

the error matrix with $k_{1}$ and $k_{2}$ as the uncertain parameters is given by

$$
E=k_{1} E_{1}+k_{2} E_{2}
$$

where

$$
E_{1}=\left[\begin{array}{lll}
1 & 0 & 1 \\
0 & 0 & 0 \\
1 & 0 & 1
\end{array}\right] \text { and } E_{2}=\left[\begin{array}{ccc}
0 & 0 & 0 \\
0 & 1 & 0 \\
0 & 1 & 0
\end{array}\right]
$$

The following are the bounds on $\left|k_{1}\right|$ and $\left|k_{2}\right|$ obtained by [15] and the proposed method.

\begin{tabular}{cccc}
$\mu_{\mathrm{y}}$ & $\mu_{\mathrm{Q}}$ & ZK $[14]$ & $\mu_{\mathrm{d}}[6]$ \\
\hline 0.815 & 0.875 & 1.55 & 1.75
\end{tabular}

\subsection{Robust control design for linear systems with structured uncertainty}

Having discussed the robustness analysis issue above, we now switch our attention to the robust control design issue. Towards this direction, we now present a linear robust control design algorithm for linear deterministic uncertain systems whose parameters vary within given bounded sets. The algorithm explicitly incorporates the structure of the uncertainty into the design procedure and utilizes the elemental perturbation bounds developed above. A linear state feedback controller is designed by parameter optimization techniques to maximize (in a given sense) the elemental perturbation bounds for robust stabilization.

There is a considerable amount of literature on the aspect of designing linear controllers for linear tine invariant systems with small parameter uncertainty. However, for uncertain systems whose dynamics are described by interval matrices (i.e., matrices whose elements are known to vary within a given bounded interval), linear control design schemes that guarantee stability have been relatively scarce. Reference [17] compares several techniques for designing linear controllers for robust stability for a class of uncertain linear systems. Among the methods considered are the standard linear quadratic regulator (LQR) design, Guaranteed Cost Control (GCC) method of [18], Multistep Guaranteed Cost Control (MGCC) of [17]. In these methods, the weighting on state in a quadratic cost function and the Riccati equation are modified in the search for an appropriate controller. Also, the parameter uncertainty is assumed to enter linearly and restrictive conditions are imposed on the bounding sets. In [18], norm inequalities on the bounding sets are given for stability but 
they are conservative since they do not take advantage of the system structure. There is no guarantee that a linear state feedback controller exists. Reference [19] utilizes the concept of 'Matching conditions (MC)' which in essence constrain the manner in which the uncertainty is permitted to enter into the dynamics and show that a linear state feedback control that guarantees stability exists provided the uncertainty satisfies matching conditions. By this method large bounding sets produce large feedback gains but the existence of a linear controller is guaranteed. But no such guarantee can be given for general 'mismatched' uncertain systems. References [20] and [21] present methods which need the testing of definiteness of a Lyapunov matrix obtained as a function of the uncertain parameters. In the multimodel theory approach, [22] considers a discrete set of points in the parameter uncertainty range to establish the stability. This paper addresses the stabilization problem for a continuous range of parameters in the uncertain parameter set (i.e. in the context of interval matrices). The proposed approach attacks the stability of interval matrix problem directly in the matrix domain rather than converting the interval matrix to interval polynomials and then testing the Kharitonov polynomials.

Robust control design using perturbation bound analysis [23],[24]

Consider a linear, time invariant system described by

$$
\dot{x}=A x+B u \quad x(0)=x_{0}
$$

Where $x$ is $n \times 1$ state vector, the control $\mathrm{u}$ is $m \times 1$. The matrix pair $(A, B)$ is assumed to be completely controllable.

$U=G x$

For this case, the nominal closed loop system matrix is given by

$$
\bar{A}=A+B G, G=-R_{0}^{-1} B^{T} K / \rho_{c}
$$

and

$$
K A+A^{T} K-K B \frac{R_{0}^{-1}}{\rho_{c}} B^{T} K+Q=0
$$

and $\bar{A}$ is asymptotically stable.

Here $G$ is the Riccati based control gain where $Q$, and $R_{0}$ are any given weighting matrices which are symmetric, positive definite and $\rho_{c}$ is the design variable.

The main interest in determining $G$ is to keep the nominal closed loop system stable. The reason Riccati approach is used to determine $G$ is that it readily renders $(A+B G)$ asymptotically stable with the above assumption on $Q$ and $R_{0}$.

Now consider the perturbed system with linear time varying perturbations $E_{A}(t)$ and $E_{B}(t)$ respectively in matrices $A$ and $B$

i.e., $\dot{x}=\left[A+E_{A}(t)\right] x(t)+\left[B+E_{B}(t)\right] u(t)$

Let $\Delta A$ and $\Delta B$ be the perturbation matrices formed by the maximum modulus deviations expected in the individual elements of matrices $A$ and $B$ respectively. Then one can write

$$
\begin{aligned}
& \Delta A=\varepsilon_{a} U_{e a} \\
& \Delta B=\varepsilon_{b} U_{e b}
\end{aligned} \text { (Absolute variation) }
$$


where $\varepsilon_{a}$ is the maximum of all the elements in $\Delta A$ and $\varepsilon_{b}$ is the maximum of all elements in $\Delta B$. Then the total perturbation in the linear closed loop system matrix of (10) with nominal control $u=G x$ is given by

$$
\Delta=\Delta A+\Delta B G_{m}=\varepsilon_{a} U_{e a}+\varepsilon_{b} U_{e b} G_{m}
$$

Assuming the ratio is $\varepsilon_{b} / \varepsilon_{a}=\bar{\varepsilon}$ known, we can extend the main result of equation (3) to the linear state feedback control system of (9) and (10) and obtain the following design observation.

\section{Design observation 1:}

The perturbed linear system is stable for all perturbations bounded by $\varepsilon_{a}$ and $\varepsilon_{b}$ if

$$
\varepsilon_{a}<\frac{1}{\sigma_{\max }\left[P_{m}\left(U_{e a}+\bar{\varepsilon} U_{e b} G_{m}\right)\right]_{s}} \equiv \mu
$$

and $\varepsilon_{b}<\bar{\varepsilon} \mu$ where

$$
P(A+B G)+(A+B G)^{T} P+2 I_{n}=0
$$

Remark: If we suppose $\Delta \mathrm{A}=0, \Delta \mathrm{B}=0$ and expect some control gain perturbations $\Delta \mathrm{G}$, where we can write

then stability is assured if

$$
\Delta G=\varepsilon_{g} U e_{g}
$$

$$
\varepsilon_{g}<\frac{1}{\sigma_{\max }\left(P_{m} B_{m} U_{e g}\right)_{s}} \equiv \mu_{g}
$$

In this context $\mu g$ can be regarded as a "gain margin".

For a given $\varepsilon_{a i j}$ and $\varepsilon_{b i j}$, one method of designing the linear controller would be to determine $\mathrm{G}$ of (3.10) by varying $\rho_{c}$ of (3.10) such that $\mu$ is maximum. For an aircraft control example which utilizes this method, see Reference [9].

\section{Robust stability and control of linear interval parameter systems using ecological perspective}

It is well recognized that natural systems such as ecological and biological systems are highly robust under various perturbations. On the other hand, engineered systems can be made highly optimal for good performance but they tend to be non-robust under perturbations. Thus, it is natural and essential for engineers to delve into the question of as to what the underlying features of natural systems are, which make them so robust and then try to apply these principles to make the engineered systems more robust. Towards this objective, the interesting aspect of qualitative stability in ecological systems is considered in particular. The fields of population biology and ecology deal with the analysis of growth and decline of populations in nature and the struggle of species to predominate over one another. The existence or extinction of a species, apart from its own effect, depends on its interactions with various other species in the ecosystem it belongs to. Hence the type of interaction is very critical to the sustenance of species. In the following sections these 
interactions and their nature are thoroughly investigated and the effect of these qualitative interactions on the quantitative properties of matrices, specifically on three matrix properties, namely, eigenvalue distribution, normality/condition number and robust stability are presented. This type of study is important for researchers in both fields since qualitative properties do have significant impact on the quantitative aspects. In the following sections, this interrelationship is established in a sound mathematical framework. In addition, these properties are exploited in the design of controllers for engineering systems to make them more robust to uncertainties such as described in the previous sections.

\subsection{Robust stability analysis using principles of ecology 4.1.1 Brief review of ecological principles}

In this section a few ecological system principles that are of relevance to this chapter are briefly reviewed. Thorough understanding of these principles is essential to appreciate their influence on various mathematical results presented in the rest of the chapter.

In a complex community composed of many species, numerous interactions take place. These interactions in ecosystems can be broadly classified as i) Mutualism, ii) Competition, iii) Commensalism/ Ammensalism and iv) Predation (Parasitism). Mutualism occurs when both species benefit from the interaction. When one species benefits/suffers and the other one remains unaffected, the interaction is classified as Commensalism/Ammensalism. When species compete with each other, that interaction is known as Competition. Finally, if one species is benefited and the other suffers, the interaction is known as Predation (Parasitism). In ecology, the magnitudes of the mutual effects of species on each other are seldom precisely known, but one can establish with certainty, the types of interactions that are present. Many mathematical population models were proposed over the last few decades to study the dynamics of eco/bio systems, which are discussed in textbooks [25][26]. The most significant contributions in this area come from the works of Lotka and Volterra. The following is a model of a predator-prey interaction where $x$ is the prey and $y$ is the predator.

$$
\begin{aligned}
& \dot{x}=x f(x, y) \\
& \dot{y}=y g(x, y)
\end{aligned}
$$

where it is assumed that $\partial f(x, y) / \partial y<0$ and $\partial g(x, y) / \partial x>0$

This means that the effect of $y$ on the rate of change of $x(\dot{x})$ is negative while the effect of $x$ on the rate of change of $\mathrm{y}(\dot{y})$ is positive.

The stability of the equilibrium solutions of these models has been a subject of intense study in life sciences [27]. These models and the stability of such systems give deep insight into the balance in nature. If a state of equilibrium can be determined for an ecosystem, it becomes inevitable to study the effect of perturbation of any kind in the population of the species on the equilibrium. These small perturbations from equilibrium can be modeled as linear state space systems where the state space plant matrix is the 'Jacobian'. This means that technically in the Jacobian matrix, one does not know the actual magnitudes of the partial derivatives but their signs are known with certainty. That is, the nature of the interaction is known but not the strengths of those interactions. As mentioned previously, there are four classes of interactions and after linearization they can be represented in the following manner. 


\begin{tabular}{|c|c|c|}
\hline Interaction type & $\begin{array}{c}\text { Digraph } \\
\text { representation }\end{array}$ & $\begin{array}{c}\text { Matrix } \\
\text { representation }\end{array}$ \\
\hline Mutualism & & {$\left[\begin{array}{ll}* & + \\
+ & *\end{array}\right]$} \\
\hline Competition & 2 & {$\left[\begin{array}{ll}* & - \\
- & *\end{array}\right]$} \\
\hline Commensalism & 2 & {$\left[\begin{array}{ll}* & + \\
0 & *\end{array}\right]$} \\
\hline Ammensalism & 1 & {$\left[\begin{array}{ll}* & - \\
0 & *\end{array}\right]$} \\
\hline $\begin{array}{c}\text { Predation } \\
\text { (Parasitism) }\end{array}$ & 2 & {$\left[\begin{array}{ll}* & + \\
- & *\end{array}\right]$} \\
\hline
\end{tabular}

Table 1. Types of interactions between two species in an ecosystem

In Table 1, column 2 is a visual representation of such interactions and is known as a directed graph or 'digraph' [28] while column 3 is the matrix representation of the interaction between two species. ' ${ }^{\prime \prime}$ represents the effect of a species on itself.

In other words, in the Jacobian matrix, the 'qualitative' information about the species is represented by the signs,+- or 0 . Thus, the $(i, j)^{\text {th }}$ entry of the state space (Jacobian) matrix simply consists of signs,+- , or 0 , with the + sign indicating species $j$ having a positive influence on species $i$, - sign indicating negative influence and 0 indicating no influence. The diagonal elements give information regarding the effect of a species on itself. Negative sign means the species is 'self-regulatory', positive means it aids the growth of its own population and zero means that it has no effect on itself. For example, in the Figure 1 below, sign pattern matrices $A_{1}$ and $A_{2}$ are the Jacobian form while $D_{1}$ and $D_{2}$ are their corresponding digraphs.

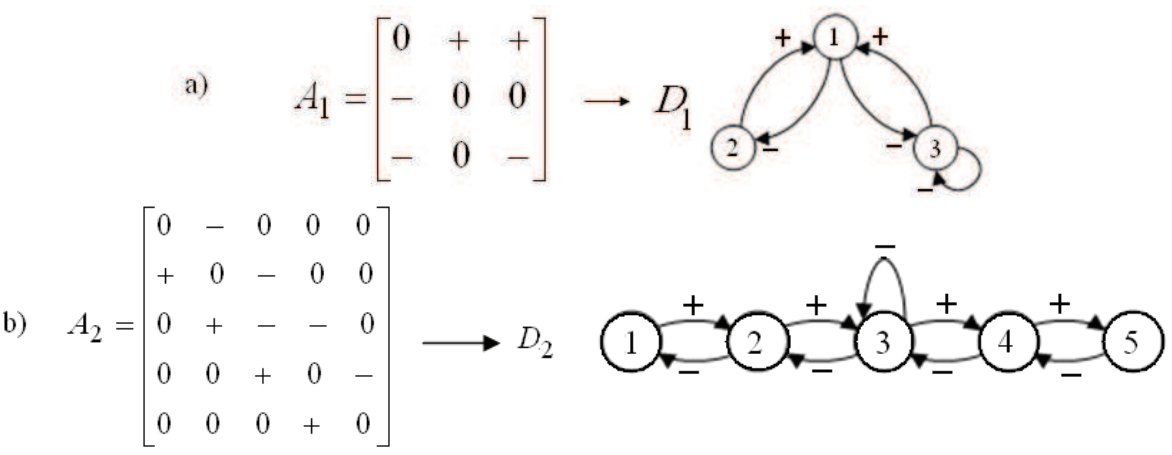

Fig. 1. Various sign patterns and their corresponding digraphs representing ecological systems; a) three species system b) five species system 


\subsubsection{Qualitative or sign stability}

Since traditional mathematical tests for stability fail to analyze the stability of such ecological models, an extremely important question then, is whether it can be concluded, just from this sign pattern, whether the system is stable or not. If so, the system is said to be 'qualitatively stable' [29-31]. In some literature, this concept is also labeled as 'sign stability'. In what follows, these two terms are used interchangeably. It is important to keep in mind that the systems (matrices) that are qualitatively (sign stable) stable are also stable in the ordinary sense. That is, qualitative stability implies Hurwitz stability (eigenvalues with negative real part) in the ordinary sense of engineering sciences. In other words, once a particular sign matrix is shown to be qualitatively (sign) stable, any magnitude can be inserted in those entries and for all those magnitudes the matrix is automatically Hurwitz stable. This is the most attractive feature of a sign stable matrix. However, the converse is not true. Systems that are not qualitatively stable can still be stable in the ordinary sense for certain appropriate magnitudes in the entries. From now on, to distinguish from the concept of 'qualitative stability' of life sciences literature, the label of 'quantitative stability' for the standard Hurwitz stability in engineering sciences is used.

These conditions in matrix theory notation are given below

i. $\quad a_{i i} \leq 0 \quad \forall i$

ii. and $a_{i i}<0$ for at least one $i$

iii. $a_{i j} a_{j i} \leq 0 \quad \forall i, j \quad i \neq j$

iv. $a_{i j} a_{j k} a_{k l} \ldots a_{m i}=0$ for any sequence of three or more distinct indices $i, j, k, \ldots m$.

v. $\operatorname{Det}(A) \neq 0$

vi. Color test (Elaborated in [32],[33])

Note: In graph theory $a_{i j} a_{j i}$ are referred to as $l$-cycles and $a_{i j} a_{j k} a_{k l} \ldots a_{m i}$ are referred to as $k$-cycles. In [34], [35], $l$-cycles are termed 'interactions' while $k$-cycles are termed 'interconnections' (which essentially are all zero in the case of sign stable matrices).

With this algorithm, all matrices that are sign stable can be stored apriori as discussed in [36]. If a sign pattern in a given matrix satisfies the conditions given in the above papers (thus in the algorithm), it is an ecological stable sign pattern and hence that matrix is Hurwitz stable for any magnitudes in its entries. A subtle distinction between 'sign stable' matrices and 'ecological sign stable' matrices is now made, emphasizing the role of nature of interactions. Though the property of Hurwitz stability is held in both cases, ecosystems sustain solely because of interactions between various species. In matrix notation this means that the nature of off-diagonal elements is essential for an ecosystem. Consider a strictly upper triangular $3 \times 3$ matrix

$$
A=\left[\begin{array}{ccc}
- & 0 & + \\
0 & - & 0 \\
0 & 0 & -
\end{array}\right]
$$

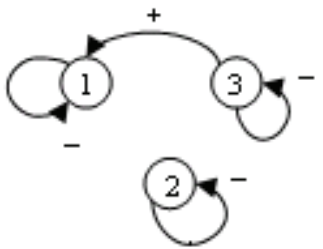

From quantitative viewpoint, it is seen that the matrix is Hurwitz stable for any magnitudes in the entries of the matrix. This means that it is indeed (qualitatively) sign stable. But since there is no predator-prey link and in fact no link at all between species $1 \& 2$ and $3 \& 2$, such a 
digraph cannot represent an ecosystem. Therefore, though a matrix is sign stable, it need not belong to the class of ecological sign stable matrices. In Figure 2 below, these various classes of sign patterns and the corresponding relationship between these classes is depicted. So, every ecological sign stable sign pattern is sign stable but the converse is not true.

With this brief review of ecological system principles, the implications of these ecological qualitative principles on three quantitative matrix theory properties, namely eigenvalues, normality/condition number and robust stability are investigated. In particular, in the next section, new results that clearly establish these implications are presented. As mentioned in the previous section, the motivation for this study and analysis is to exploit some of these desirable features of ecological system principles to design controllers for engineering systems to make them more robust.

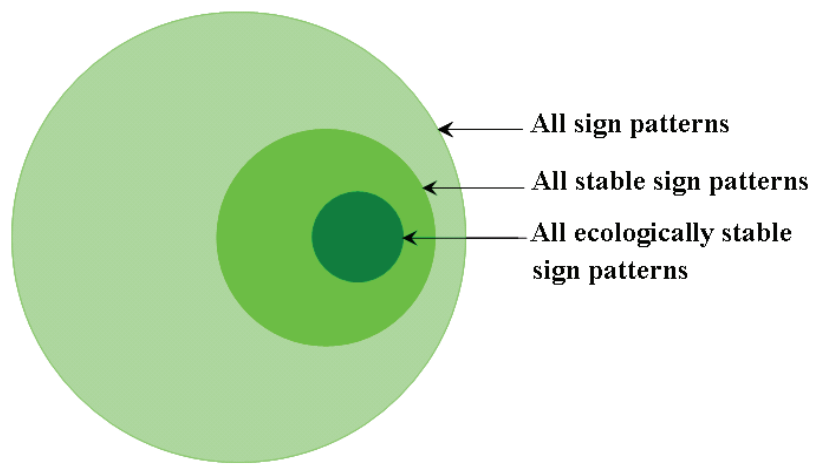

Fig. 2. Classification of sign patterns

\subsection{Ecological sign stability and its implications in quantitative matrix theory}

In this major section of this chapter, focusing on the ecological sign stability aspect discussed above, its implications in the quantitative matrix theory are established. In particular, the section offers three explicit contributions to expand the current knowledge base, namely i) Eigenvalue distribution of ecological sign stable matrices ii) Normality/Condition number properties of sign stable matrices and iii) Robustness properties of sign stable matrices. These three contributions in turn help in determining the role of magnitudes in quantitative ecological sign stable matrices. This type of information is clearly helpful in designing robust controllers as shown in later sections. With this motivation, a 3-species ecosystem is thoroughly analyzed and the ecological principles in terms of matrix properties that are of interest in engineering systems are interpreted. This section is organized as follows: First, new results on the eigenvalue distribution of ecological sign stable matrices are presented. Then considering ecological systems with only predation-prey type interactions, it is shown how selection of appropriate magnitudes in these interactions imparts the property of normality (and thus highly desirable condition numbers) in matrices. In what follows, for each of these cases, concepts are first discussed from an ecological perspective and then later the resulting matrix theory implications from a quantitative perspective are presented Stability and eigenvalue distribution

Stability is the most fundamental property of interest to all dynamic systems. Clearly, in time invariant matrix theory, stability of matrices is governed by the negative real part 
nature of its eigenvalues. It is always useful to get bounds on the eigenvalue distribution of a matrix with as little computation as possible, hopefully as directly as possible from the elements of that matrix. It turns out that sign stable matrices have interesting eigenvalue distribution bounds. A few new results are now presented in this aspect.

In what follows, the quantitative matrix theory properties for an n-species ecological system is established, i.e., an $\mathrm{n} \times \mathrm{n}$ sign stable matrix with predator-prey and commensal/ammensal interactions is considered and its eigenvalue distribution is analyzed. In particular, various cases of diagonal elements' nature, which are shown to possess some interesting eigenvalue distribution properties, are considered.

\section{Bounds on real part of eigenvalues}

Based on several observations the following theorem for eigenvalue distribution along the real axis is stated.

Theorem 1 [37]

(Case of all negative diagonal elements):

For all $n \times n$ sign stable matrices, with all negative diagonal elements, the bounds on the real parts of the eigenvalues are given as follows:

The lower bound on the magnitude of the real part is given by the minimum magnitude diagonal element and the upper bound is given by the maximum magnitude diagonal element in the matrix.

That is, for an $n \times n$ ecological sign stable matrix $A=\left[a_{i j}\right]$,

$$
\left|a_{i i}\right|_{\min } \leq|\operatorname{Re}(\lambda)|_{\min } \leq|\operatorname{Re}(\lambda)|_{\max } \leq\left|a_{i i}\right|_{\max }
$$

Corollary

(Case of some diagonal elements being zero):

If the ecological sign stable matrix has zeros on the diagonal, the bounds are given by

$$
\left|a_{i i}\right|_{\min }(=0)<|\operatorname{Re}(\lambda)|_{\min } \leq|\operatorname{Re}(\lambda)|_{\max } \leq\left|a_{i i}\right|_{\max }
$$

The sign pattern in Example 1 has all negative diagonal elements. In this example, the case discussed in the corollary where one of the diagonal elements is zero, is considered. This sign pattern is as shown in the matrix below.

$$
A=\left[\begin{array}{lll}
- & - & - \\
0 & - & 0 \\
+ & + & 0
\end{array}\right]
$$

\section{Bounds on imaginary part of eigenvalues [38]}

Similarly, the following theorem can be stated for bounds on the imaginary parts of the eigenvalues of an $\mathrm{n} \times \mathrm{n}$ matrix. Before stating the theorem, we present the following lemma.

Theorem 2

For all $n \times n$ ecologically sign stable matrices, bound on the imaginary part of the eigenvalues is given by

$$
\left.\mu\right|_{\text {imagss }}=\left|\operatorname{Imag}\left(\lambda_{\mathrm{i}}\right)\right|_{\max }=\sqrt{\sum_{i, j=1}^{n}-a_{i j} a_{j i}} \quad \forall i \neq j
$$

Above results are illustrated in figure 3. 


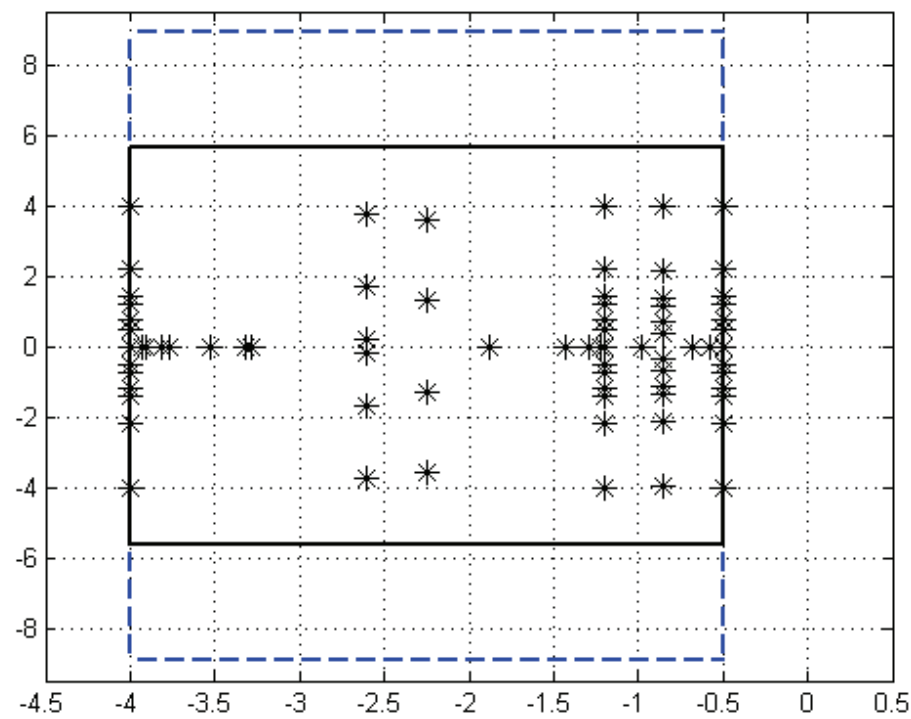

Fig. 3. Eigenvalue distribution for sign stable matrices

Theorem 3

For all $n \times n$ matrices, with all $k$-cycles being zero and with only commensal or ammensal interactions, the eigenvalues are simply the diagonal elements.

It is clear that these theorems offer significant insight into the eigenvalue distribution of $n \times n$ ecological sign stable matrices. Note that the bounds can be simply read off from the magnitudes of the elements of the matrices. This is quite in contrast to the general quantitative Hurwitz stable matrices where the lower and upper bounds on the eigenvalues of a matrix are given in terms of the singular values of the matrix and/or the eigenvalues of the symmetric part and skew-symmetric parts of the matrices (using the concept of field of values), which obviously require much computation, and are complicated functions of the elements of the matrices.

Now label the ecological sign stable matrices with magnitudes inserted in the elements as 'quantitative ecological sign stable matrices'. Note that these magnitudes can be arbitrary in each non zero entry of the matrix! It is interesting and important to realize that these bounds, based solely on sign stability, do not reflect diagonal dominance, which is the typical case with general Hurwitz stable matrices. Taking theorems 4, 5, 6 and their respective corollaries into consideration, we can say that it is the 'diagonal connectance' that is important in these quantitative ecological sign stable matrices and not the 'diagonal dominance' which is typical in the case of general Hurwitz stable matrices. This means that interactions are critical to system stability even in the case of general $n \times n$ matrices.

Now the effect on the quantitative property of normality is presented.

\section{Normality and condition number}

Based on this new insight on the eigenvalue distribution of sign stable matrices, other matrix theory properties of sign stable matrices are investigated. The first quantitative matrix theory property is that of normality/condition number. But this time, the focus is only on ecological sign stable matrices with pure predator-prey links with no other types of interactions. 
A zero diagonal element implies that a species has no control over its growth/decay rate. So in order to regulate the population of such a species, it is essential that, in a sign stable ecosystem model, this species be connected to at least one predator-prey link. In the case where all diagonal elements are negative, the matrix represents an ecosystem with all selfregulating species. If every species has control over its regulation, a limiting case for stability is a system with no interspeciel interactions. This means that there need not be any predator-prey interactions. This is a trivial ecosystem and such matrices actually belong to the only 'sign-stable' set, not to ecological sign stable set.

Apart from the self-regulatory characteristics of species, the phenomena that contribute to the stability of a system are the type of interactions. Since a predator-prey interaction has a regulating effect on both the species, predator-prey interactions are of interest in this stability analysis. In order to study the role played by these interactions, henceforth focus is on systems with n-1 pure predator-prey links in specific places. This number of links and the specific location of the links are critical as they connect all species at the same time preserving the property of ecological sign stability. For a matrix $A$, pure predator-prey link structure implies that

1. $A_{i j} A_{j i} \leq 0 \quad \forall i, j$

2. $A_{i j} A_{j i}=0$ iff $A_{i j}=A_{j i}=0$

Hence, in what follows, matrices with all negative diagonal elements and with pure predator-prey links are considered.

Consider sign stable matrices with identical diagonal elements (negative) and pure predator-prey links of equal strengths.

Normality in turn implies that the modal matrix of the matrix is orthogonal resulting in it having a condition number of one, which is an extremely desirable property for all matrices occurring in engineering applications.

The property of normality is observed in higher order systems too. An ecologically sign stable matrix with purely predator-prey link interactions is represented by the following digraph for a 5 -species system. The sign pattern matrix $A$ represents this digraph.

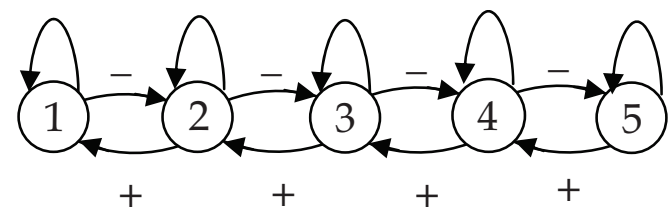

$$
A=\left[\begin{array}{lllll}
- & + & 0 & 0 & 0 \\
- & - & + & 0 & 0 \\
0 & - & - & + & 0 \\
0 & 0 & - & - & + \\
0 & 0 & 0 & - & -
\end{array}\right]
$$

\section{Theorem 4}

An $n \times n$ matrix $A$ with equal diagonal elements and equal predation prey interaction strengths for each predation-prey link is a normal matrix.

The property of $\kappa \equiv 1$ is of great significance in the study of robustness of stable matrices. This significance will be explained in the next section eventually leading to a robust control design algorithm

\section{Robustness}

The third contribution of this section is related to the connection between ecological sign stability and robust stability in engineering systems. 
As mentioned earlier, the most interesting feature of ecological sign stable matrices is that the stability property is independent of the magnitude information in the entries of the matrix. Thus the nature of interactions, which in turn decide the signs of the matrix entries and their locations in the matrix, are sufficient to establish the stability of the given sign matrix. Clearly, it is this independence (or non-dependence) from magnitude information that imparts the property of robust stability to engineering systems. This aspect of robust stability in engineering systems is elaborated next from quantitative matrix theory point of view.

\section{Robustness as a result of independence from magnitude information}

In mathematical sciences, the aspect of 'robust stability' of families of matrices has been an active topic of research for many decades. This aspect essentially arises in many applications of system and control theory. When the system is described by linear state space representation, the plant matrix elements typically depend on some uncertain parameters which vary within a given bounded interval.

Robust stability analysis of a class of interval matrices [39]:

Consider the 'interval matrix family' in which each individual element varies independently within a given interval. Thus the interval matrix family is denoted by

$A \in\left[A^{L}, A^{U}\right]$ as the set of all matrices $A$ that satisfy

$$
\left(A^{L}\right)_{i j} \leq A_{i j} \leq\left(A^{U}\right)_{i j} \text { for every } i, j
$$

Now, consider a special 'class of interval matrix family' in which for each element that is varying, the lower bound i.e. $\left(A^{L}\right)_{i j}$ and the upper bound i.e. $\left(A^{U}\right)_{i j}$ are of the same sign.

For example, consider the interval matrix given by

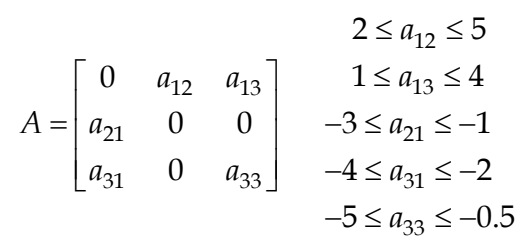

with the elements $a_{12}, a_{13}, a_{21}, a_{31}$ and $a_{33}$ being uncertain varying in some given intervals as follows:

Qualitative stability as a 'sufficient condition' for robust stability of a class of interval matrices: A link between life sciences and engineering sciences

It is clear that ecological sign stable matrices have the interesting feature that once the sign pattern is a sign stable pattern, the stability of the matrix is independent of the magnitudes of the elements of the matrix. That this property has direct link to stability robustness of matrices with structured uncertainty was recognized in earlier papers on this topic [32] and [33]. In these papers, a viewpoint was put forth that advocates using the 'qualitative stability' concept as a means of achieving 'robust stability' in the standard uncertain matrix theory and offer it as a 'sufficient condition' for checking the robust stability of a class of interval matrices. This argument is illustrated with the following examples.

Consider the above given 'interval matrix'.

Once it is recognized that the signs of the interval entries in the matrix are not changing (within the given intervals), the sign matrix can be formed. The 'sign' matrix for this interval matrix is given by 


$$
A=\left[\begin{array}{lll}
0 & + & + \\
- & 0 & 0 \\
- & 0 & -
\end{array}\right]
$$

The above 'sign' matrix is known to be 'qualitative (sign) stable'. Since sign stability is independent of magnitudes of the entries of the matrix, it can be concluded that the above interval matrix is robustly stable in the given interval ranges. Incidentally, if the 'vertex algorithm' of [40] is applied for this problem, it can be also concluded that this 'interval matrix family' is indeed Hurwitz stable in the given interval ranges.

In fact, more can be said about the 'robust stability' of this matrix family using the 'sign stability' application. This matrix family is indeed robustly stable, not only for those given interval ranges above, but it is also robustly stable for any large 'interval ranges' in those elements as long as those interval ranges are such that the elements do not change signs in those interval ranges. In the above discussion, the emphasis was on exploiting the sign pattern of a matrix in robust stability analysis of matrices. Thus, the tolerable perturbations are direction sensitive. Also, no perturbation is allowed in the structural zeroes of the ecological sign stable matrices. In what follows, it is shown that ecological sign stable matrices can still possess superior robustness properties even under norm bounded perturbations, in which perturbations in structural zeroes are also allowed in ecological sign stable matrices.

Towards this objective, the stability robustness measures of linear state space systems as discussed in [39] and [2] are considered. In other words, a linear state space plant matrix $A$, which is assumed to be Hurwitz stable, is considered. Then assuming a perturbation matrix $E$ in the $A$ matrix, the question as to how much of norm of the perturbation matrix $E$ can be tolerated to maintain stability is asked. Note that in this norm bounded perturbation discussion, the elements of the perturbation matrix can vary in various directions without any restrictions on the signs of the elements of that matrix. When bounds on the norm of $E$ are given to maintain stability, it is labeled as robust stability for unstructured, norm bounded uncertainty. We now briefly recall two measures of robustness available in the literature [2] for robust stability of time varying real parameter perturbations.

Norm bounded robustness measures

Consider a given Hurwitz stable matrix $A_{0}$ with perturbation $E$ such that

$$
A=A_{0}+E
$$

where $A$ is any one of the perturbed matrices.

A sufficient bound $\mu$ for the stability of the perturbed system is given on the spectral norm of the perturbation matrix as

$$
\|E\|<-\frac{\alpha_{s}}{\kappa}=\frac{\left|\operatorname{Re}\left(\lambda\left(A_{0}\right)\right)\right|_{\min }}{\kappa}=\mu_{d}
$$

where $\alpha_{s}$ is the real part of the dominant eigenvalue, also known as stability degree and $\kappa$ is the condition number of the modal matrix of $A_{0}$.

Theorem 5[38]

$$
\begin{aligned}
& \left|\operatorname{Re}\left(\lambda\left(A_{N N}\right)\right)\right|_{\min }>\left|\operatorname{Re}\left(\lambda\left(B_{N N}\right)\right)\right|_{\min } \\
& \text { i.e., } \mu\left(A_{N N}\right)>\mu\left(B_{N N}\right)
\end{aligned}
$$


In other words, a unit norm, normal ecological sign stable matrix is more robust that a unit norm, normal non-ecological sign stable Hurwitz stable matrix.

The second norm bound based on the solution of the Lyapunov matrix equation [7] is given as

$$
\|E\|<\mu_{p}=\frac{1}{\sigma_{\max }(P)}
$$

where

$P$ is the solution of the Lyapunov equation of the nominal stable matrix $A_{0}$ given by

$$
A_{0}^{T} P+P A_{0}+2 I=0
$$

Based on this bound, the following Lemma is proposed:

Theorem 6

The norm bound $\mu_{p}$ on a target SS matrix $S$ is $d$, where $d$ is the magnitude of diagonal element of $S$ i.e.,

$$
\mu_{p}=\frac{1}{\sigma_{\max }(P)}=d
$$

This means that for any given value of $\mu_{p}$, we can, by mere observation, determine a corresponding stable matrix A!

This gives impetus to design controllers that drive the closed loop system to a target matrix. Towards this objective, an algorithm for the design of a controller based on concepts from ecological sign stability is now presented.

\subsection{Robust control design based on ecological sign stability}

Extensive research in the field of robust control design has lead to popular control design methods in frequency domain such as $H_{\infty}$ and $\mu$-synthesis., Though these methods perform well in frequency domain, they become very conservative when applied to the problem of accommodating real parameter uncertainty. On the other hand, there are very limited robust control design methods in time domain methods that explicitly address real parameter uncertainty [41-47]. Even these very few methods tend to be complex and demand some specific structure to the real parameter uncertainty (such as matching conditions). Therefore, as an alternative to existing methods, the distinct feature of this control design method inspired by ecological principles is its problem formulation in which the robustness measure appears explicitly in the design methodology.

\subsubsection{Problem formulation}

The problem formulation for this novel control design method is as follows:

For a given linear system

$$
\dot{x}(t)=A x(t)+B u(t)
$$

design a full-state feedback controller

$$
u=G x
$$


where the closed loop system

$$
A_{n \times n}+B_{n \times m} G_{m \times n}=A_{c l n \times n}
$$

possesses a desired robustness bound $\mu$ (there is no restriction on the value this bound can assume).

Since eigenvalue distribution, condition number (normality) and robust stability properties have established the superiority of target matrices, they become an obvious choice for the closed loop system matrix $A_{c l}$. Note that the desired bound $\mu=\mu_{d}=\mu_{p}$. Therefore, the robust control design method proposed in the next section addresses the three viewpoints of robust stability simultaneously!

\subsubsection{Robust control design algorithm}

Consider the LTI system

$$
\dot{x}=A x+B u
$$

Then, for a full-state feedback controller, the closed loop system matrix is given by

$$
\begin{aligned}
& A_{n x n}+B_{n x m} G_{m x n}=A_{c l n x n}\left(=A_{t}\right) \\
& \text { Let } A_{c l}-A=A_{a}
\end{aligned}
$$

The control design method is classified as follows:

1. Determination of Existence of the Controller[38]

2. Determination of Appropriate Closed loop System[38]

3. Determination of Control Gain Matrix[48]

Following example illustrates this simple and straightforward control design method.

Application: Satellite formation flying control problem

The above control algorithm is now illustrated for the application discussed in [32],[33] and [49].

$$
\left[\begin{array}{c}
\dot{x} \\
\ddot{x} \\
\dot{y} \\
\ddot{y}
\end{array}\right]=\left[\begin{array}{cccc}
0 & 0 & 1 & 0 \\
0 & 0 & 0 & 1 \\
0 & 0 & 0 & 2 \omega \\
0 & 3 \omega^{2} & -2 \omega & 0
\end{array}\right]\left[\begin{array}{c}
x \\
\dot{x} \\
y \\
\dot{y}
\end{array}\right]+\left[\begin{array}{ll}
0 & 0 \\
0 & 0 \\
1 & 0 \\
0 & 1
\end{array}\right]\left[\begin{array}{l}
T_{x} \\
T_{y}
\end{array}\right]
$$

where $x, \dot{x}, y$ and $\dot{y}$ are the state variables, $T_{x}$ and $T_{y}$ are the control variables.

For example, when $\omega=1$, the system becomes

$$
A=\left[\begin{array}{cccc}
0 & 0 & 1 & 0 \\
0 & 0 & 0 & 1 \\
0 & 0 & 0 & 2 \\
0 & 3 & -2 & 0
\end{array}\right] \text { and } B=\left[\begin{array}{ll}
0 & 0 \\
0 & 0 \\
1 & 0 \\
0 & 1
\end{array}\right]
$$

Clearly, the first two rows of $A_{c l}$ cannot be altered and hence a target matrix with all nonzero elements cannot be achieved. Therefore, a controller such that the closed loop system has as many features of a target SS matrix as possible is designed as given below. 
Accordingly, an ecological sign stable closed loop system is chosen such that

i The closed loop matrix has as many pure predator-prey links as possible.

ii It also has as many negative diagonal elements as possible.

Taking the above points into consideration, the following sign pattern is chosen which is appropriate for the given $A$ and $B$ matrices:

$$
A_{c_{s s}}=\left[\begin{array}{cccc}
0 & 0 & + & 0 \\
0 & 0 & 0 & + \\
- & 0 & - & + \\
0 & - & - & -
\end{array}\right] \quad A_{c l}=\left[\begin{array}{cccc}
0 & 0 & 1 & 0 \\
0 & 0 & 0 & 1 \\
-1 & 0 & -1 & 2 \\
0 & -1 & -2 & -1
\end{array}\right]
$$

The magnitudes of the entries of the above sign matrix are decided by the stability robustness analysis theorem discussed previously i.e.,

i All non-zero $a_{i i}$ are identical.

ii $a_{i j}=-a_{j i}$ for all non-zero $a_{i j}$ else $a_{i j}=a_{j i}=0$

Hence, all the pure predator-prey links are of equal interaction strengths and the non-zero diagonal elements have identical self-regulatory intensities. Using the algorithm given above, the gain matrix is computed as shown below.

From the algorithm,

$$
G_{e S}=\left[\begin{array}{cccc}
-1.0 & 0 & -1.0 & 0 \\
0 & -4.0 & 0 & -1.0
\end{array}\right]
$$

The closed loop matrix $A_{c l}\left(=A+B G_{e s}\right)$ is sign-stable and hence can tolerate any amount of variation in the magnitudes of the elements with the sign pattern kept constant.

In this application, it is clear that all non-zero elements in the open loop matrix (excluding elements $A_{13}$ and $A_{24}$ since they are dummy states used to transform the system into a set of first order differential equations) are functions of the angular velocity $\omega$. Hence, real life perturbations in this system occur only due to variation in angular velocity $\omega$. Therefore, a perturbed satellite system is simply an $A$ matrix generated by a different $\omega$. This means that not every randomly chosen matrix represents a physically perturbed system and that for practical purposes, stability of the matrices generated as mentioned above (by varying $\omega$ ) is sufficient to establish the robustness of the closed loop system. It is only because of the ecological perspective that these structural features of the system are brought to light. Also, it is the application of these ecological principles that makes the control design for satellite formation flying this simple and insightful.

Ideally, we would like $A_{t}$ to be the eventual closed loop system matrix. However, it may be difficult to achieve this objective for any given controllable pair $(A, B)$. Therefore, we propose to achieve a closed loop system matrix that is close to $A_{t}$. Thus the closed loop system is expressed as

$$
A_{c l}=A+B G=A_{t}+\Delta A
$$

Noting that ideally we like to aim for $\Delta A=0$, we impose this condition. Then, $A_{c l}=A_{t}=$ $A+B G$.

i. When $B$ is square and invertible: As given previously,

$$
A_{c l}=A_{t} \text { and } G=B^{-1}\left(A-A_{t}\right)
$$


ii. When $B$ is not square, but has full rank:

Consider $B^{\dagger}$, the pseudo inverse of $B$

where, for $B_{\mathrm{n} \times \mathrm{m}}$, if $\mathrm{n}>\mathrm{m}, B^{\dagger}=\left(B^{T} B\right)^{-1} B^{T}$

Then $G=B^{\dagger}\left(A-A_{t}\right)$

Because of errors associated with pseudo inverse operation, the expression for the closed loop system is as follows [34]:

$$
\begin{gathered}
A_{t}+\Delta E=A+B G \\
A_{t}+\Delta E=A+B\left(B^{T} B\right)^{-1} B^{T}\left(A_{t}-A\right)
\end{gathered}
$$

Let $B\left(B^{T} B\right)^{-1} B^{T}=B_{a u g}$

Then $\Delta E=\left(A-A_{t}\right)+B_{\text {aug }}\left(A_{t}-A\right)=-\left(A_{t}-A\right)+B_{\text {aug }}\left(A_{t}-A\right)=\left(B_{\text {aug }}-I\right)\left(A_{t}-A\right)$

$$
\therefore \Delta E=\left(B_{\text {aug }}-I\right)\left(A-A_{t}\right)
$$

which should be as small as possible. Therefore, the aim is to minimize the norm of $\Delta E$. Thus, for a given controllable pair $(A, B)$, we use the elements of the desired closed loop matrix $A_{t}$ as design variables to minimize the norm of $\Delta E$.

We now apply this control design method to aircraft longitudinal dynamics problem.

\section{Application: Aircraft flight control}

Consider the following short period mode of the longitudinal dynamics of an aircraft [50].

$$
A=\left[\begin{array}{cc}
-0.334 & 1 \\
-2.52 & -0.387
\end{array}\right] \quad B=\left[\begin{array}{c}
-0.027 \\
-2.6
\end{array}\right]
$$

\begin{tabular}{|c|c|c|c|}
\hline & Open loop $A$ & Target matrix $A_{t}$ & Close loop $A_{c l}$ \\
\hline Matrix & {$\left[\begin{array}{cc}-0.334 & 1 \\
-2.52 & -0.387\end{array}\right]$} & {$\left[\begin{array}{cc}-0.3181 & 1.00073 \\
-1.00073 & -0.3181\end{array}\right]$} & {$\left[\begin{array}{cc}-0.3182 & 1.00073 \\
-1.00073 & -0.319\end{array}\right]$} \\
\hline \multirow{2}{*}{ Eigenvalues } & {$\left[\begin{array}{l}-0.3605+j 1.5872 \\
-0.3605-j 1.5872\end{array}\right]$} & {$\left[\begin{array}{l}-0.3181+j 1.00073 \\
-0.3181-j 1.00073\end{array}\right]$} & {$\left[\begin{array}{l}-0.31816+j 1.000722 \\
-0.31816-j 1.000722\end{array}\right]$} \\
\hline Norm bound & 0.2079 & 0.3181 & 0.3181426 \\
\hline
\end{tabular}

The open loop matrix properties are as follows:

Note that the open loop system matrix is stable and has a Lyapunov based robustness bound $\mu_{o p}=0.2079$.

Now for the above controllable pair $(A, B)$, we proceed with the proposed control design procedure discussed before, with the target PS matrix $A_{t}$ elements as design variables, which very quickly yields the following results:

$A_{t}$ is calculated by minimizing the norm of $\sigma_{\max }(\Delta E)$. 
Here $\sigma_{\max }(\Delta E)=1.2381 \times 10^{-4}$

For this value, following are the properties of the target matrix.

From the expression for $G$, we get

$$
G=\left[\begin{array}{ll}
-0.5843 & -0.0265
\end{array}\right]
$$

With this controller, the closed loop matrix $A_{c l}$ is determined.

It is easy to observe that the eventual closed loop system matrix is extremely close to the target $P S$ matrix (since $\sigma_{\max }(\Delta E) \approx 0$ ) and hence the resulting robustness bounds can be simply read off from the diagonal elements of the target $S S$ matrix, which in this example is also equal to the eventual closed loop system matrix. As expected, this robustness measure of the closed loop system is appreciably greater than the robustness measure of the open loop system.

This robust controller methodology thus promises to be a desirable alternative to the other robustness based controllers encompassing many fields of application.

\section{Conclusions and future directions}

In this book chapter, robust control theory is presented essentially from a state space perspective. We presented the material in two distinct parts. In the first part of the chapter, robust control theory is presented from a quantitative (engineering) perspective, making extensive use of state space models of dynamic systems. Both robust stability analysis as well as control design were addressed and elaborated. Robust stability analysis involved studying and quantifying the tolerable bounds for maintaining the stability of a nominally stable dynamic system. Robust control design dealt with the issue of synthesizing a controller to keep the closed loop systems stable under the presence of a given set of perturbations. This chapter focused on characterizing the perturbations essentially as 'real parameter' perturbations and all the techniques presented accommodate this particular modeling error. In the second part of the chapter, robustness is treated from a completely new perspective, namely from concepts of Population (Community) Ecology, thereby emphasizing the 'qualitative' nature of the stability robustness problem. In this connection, the analysis and design aspects were directed towards studying the role of 'signs' of the elements of the state space matrices in maintaining the stability of the dynamic system. Thus the concept of 'sign stability' from the field of ecology was brought out to the engineering community. This concept is relatively new to the engineering community. The analysis and control design for engineering systems using ecological principles as presented in this chapter is deemed to spur exciting new research in this area and provide new directions for future research. In particular, the role of 'interactions and interconnections' in engineering dynamic systems is shown to be of paramount importance in imparting robustness to the system and more research is clearly needed to take full advantage of these promising ideas. This research is deemed to pave the way for fruitful collaboration between population (community) ecologists and control systems engineers.

\section{References}

[1] Dorato, P., “Robust Control”, IEEE Press, New York, N.Y., 1987

[2] Dorato, P., and Yedavalli, R. K., (Eds) Recent Advances in Robust Control, IEEE Press, 1991, pp. 109-111. 
[3] Skelton, R. “Dynamic Systems Control," John Wiley and Sons, New York, 1988

[4] Barmish, B. R., "New Tools for Robustness of Linear Systems", Macmillan Publishing Company, New York, 1994

[5] Bhattacharya, S. P., Chapellat, H., and Keel, L. H., "Robust Control: The Parameteric Approach", Prentice Hall, 1995

[6] Yedavalli, R. K., "Robust Control of Uncertain Dynamic Systems: A Linear State Space Approach", Springer, 2011 (to be published).

[7] Patel, R.V. and Toda, M., "Quantitative Measures of Robustness for Multivariable Systems," Proceedings of Joint Automatic Control Conference, TP8-A, 1980.

[8] Yedavalli, R.K., Banda, S.S., and Ridgely, D.B., "Time Domain Stability Robustness Measures for Linear Regulators," AIAA Journal of Guidance, Control and Dynamics, pp. 520-525, July-August 1985.

[9] Yedavalli, R.K. and Liang, Z., "Reduced Conservation in Stability Robustness Bounds by State Transformation," IEEE Transactions on Automatic Control, Vol. AC-31, pp. 863-866, September 1986.

[10] Hyland, D.C., and Bernstein, D.S., "The Majorant Lyapunov Equations: A Nonnegative Matrix Equation for Robust Stability and Performance of Large Scale Systems," IEEE Transactions on Automatic Control, Vol. AC-32, pp. 1005-1013, November 1987.

[11] Yedavalli, R.K., "Improved Measures of Stability-Robustness for Linear State Space Models," IEEE Transactions on Automatic Control, Vol. AC-30, pp. 577-579, June 1985.

[12] Yedavalli, R.K., "Perturbation Bounds for Robust Stability in Linear State Space Models," International Journal of Control, Vol. 42, No. 6, pp. 1507-1517, 1985.

[13] Qiu, L. and Davison, E.J., "New Perturbation Bounds for the Robust Stability of Linear State Space Models," Proceedings of the 25th Conference on Decision and Control, Athens, Greece, pp. 751-755, 1986.

[14] Hinrichsen, D. and Pritchard, A. J., "Stability Radius for Structured Perturbations and the Algebraic Riccati Equation", Systems and Control Letters, Vol. 8, pp: 105-113, 198.

[15] Zhou, K. and Khargonekar, P., "Stability Robustness Bounds for Linear State Space models with Structured Uncertainty," IEEE Transactions on Automatic Control, Vol. AC-32, pp. 621-623, July 1987.

[16] Tesi, A. and Vicino, A., "Robustness Analysis for Uncertain Dynamical Systems with Structured Perturbations," Proceedings of the International Workshop on 'Robustness in Identification and Control,' Torino, Italy, June 1988.

[17] Vinkler, A. and Wood, L. J., “A comparison of General techniques for designing Controllers of Uncertain Dynamics Systems", Proceedings of the Conference on Decision and Control, pp: 31-39, San Diego, 1979

[18] Chang, S. S. L. and Peng, T. K. C., "Adaptive Guaranteed Cost Control of Systems with Uncertain Parameters," IEEE Transactions on Automatic Control, Vol. AC-17, Aug. 1972

[19] Thorp, J. S., and Barmish B. R., “On Guaranteed Stability of Uncertain Linear Systems via Linear Control”, Journal of Optimization Theory and Applications, pp: 559-579, December 1981. 
[20] Hollot, C. V. and Barmish, B. R., “Optimal Quadratic Stabilizability of Uncertain Linear Systems" Proceedings of 18 th Allerton Conference on Communication, Control and Computing, University of Illinois, 1980.

[21] Schmitendorf, W. E., “A design methodology for Robust Stabilizing Controllers”, AIAA Guidance and Control Conference, West Virginia, 1986

[22] Ackermann, J., "Multimodel Approaches to Robust Control Systems Design", IFAC Workshop on Model Error Concepts and Compensations, Boston, 1985.

[23] Yedavalli, R. K., "Time Domain Control Design for Robust Stability of Linear Regulators: Applications to Aircraft Control", Proceedings of the American control Conference, 914-919, Boston 1985.

[24] Yedavalli, R. K., “Linear Control design for Guaranteed Stability of Uncertain Linear Systems", Proceedings of the American control Conference, 990-992, Seattle, 1986.

[25] Leah Edelstein-Keshet., Mathematical models in Biology, McGraw Hill, 1988. pp. 234-236.

[26] Hofbauer, J., and Sigmund, K., "Growth Rates and Ecological Models: ABC on ODE," The Theory of Evolutions and Dynamical Systems, Cambridge University Press, London, 1988, pp. 29-59.

[27] Dambacher, J. M., Luh, H-K., Li, H. W., and Rossignol, P. A., "Qualitative Stability and Ambiguity in Model Ecosystems," The American Naturalist, Vol. 161, No. 6, June 2003, pp. 876-888.

[28] Logofet, D., Matrices and Graphs: Stability Problems in Mathematical Ecology, CRC Press, Boca Raton, 1992R, pp. 1-35.

[29] Quirk, J., and Ruppert. R., "Qualitative Economics and the Stability of Equilibrium," Reviews in Economic Studies, 32, 1965, pp. 311-326.

[30] May. R., Stability and Complexity in Model Ecosystems, Princeton University Press, Princeton, N.J., 1973, pp. 16-34.

[31] Jeffries, C., "Qualitative Stability and Digraphs in Model Ecosystems," Ecology, Vol. 55, 1974, pp. 1415-1419.

[32] Yedavalli, R. K., “Qualitative Stability Concept from Ecology and its Use in the Robust Control of Engineering Systems," Proceedings of the American Control Conference, Minneapolis, June 2006, pp. 5097-5102.

[33] Yedavalli, R. K., "New Robust and Non-Fragile Control Design Method for Linear Uncertain Systems Using Ecological Sign Stability Approach," Proceedings of the International Conference on Advances in Control and Optimisation of Dynamical Systems. (ACODS07), Bangalore, India, 2007, pp. 267-273.

[34] Devarakonda, N. and Yedavalli, R. K., "A New Robust Control design for linear systems with norm bounded time varying real parameter uncertainty", Proceedings of ASME Dynamic Systems and Controls Conference, Boston, MA, 2010.

[35] Devarakonda, N. and Yedavalli, R. K., "A New Robust Control design for linear systems with norm bounded time varying real parameter uncertainty", IEEE Conference on Decision and Control, Atlanta, GA, 2010.

[36] Yedavalli, R. K., “Robust Control Design for Linear Systems Using an Ecological Sign Stability Approach," AIAA Journal of Guidance, Control and Dynamics, Vol. 32, No. 1, Jan-Feb, 2009, pp 348-352.

[37] Yedavalli, R. K. and Devarakonda, N. "Sign Stability Concept of Ecology for Control Design with Aerospace Applications", AIAA Journal of Guidance Control and Dynamics, Vol. 33, No. 2, pp. 333-346, 2010. 
[38] Devarakonda, N. and Yedavalli, R. K., “Engineering Perspective of Ecological Sign Stability and its Application in Control Design", Proceedings of American Control Conference, Baltimore, MD, July, 2010.

[39] Yedavalli, R. K., "Flight Control Application of New Stability Robustness Bounds for Linear Uncertain Systems," Journal of Guidance, Control and Dynamics, Vol. 16, No. 6, Nov.-Dec. 1993.

[40] Yedavalli, R. K., "Robust Stability of Linear Interval Parameter Matrix Family Problem Revisited with Accurate Representation and Solution," Proceedings of American Automatic Control Conference, June, 2009, pp 3710-3717

[41] Yedavalli, R. K., 1989. "Robust Control Design for Aerospace Applications". IEEE Transactions on Aerospace and Electronic Systems, 25(3), pp. 314

[42] Keel, L. H., Bhattacharya, S. P., and Howze, J. W., 1988. "Robust Control with Structured Perturbations", IEEE Transactions on Automatic Control, AC-33(1), pp. 68

[43] Zhou, K., and P. Khargonekar, 1988. "Robust Stabilization of Linear Systems with Norm Bounded Time Varying Uncertainty", Systems and Control Letters, 8, pp. 17.

[44] Petersen, I, R., and Hollot, C. V., 1986. "A Riccati Equation Based Approach to the Stabilization of Uncertain Linear Systems", Automatica, 22(4), pp. 397.

[45] Petersen, I, R., 1985. “A Riccati Equation Approach to the Design of Stabilizing Controllers and Observers for a Class of Uncertain Linear Systems", IEEE Transactions AC-30(9)

[46] Barmish, B. R., Corless, M., and Leitmann, G., 1983. “A New Class of Stabilizing Controllers for Uncertain Dynamical Systems", SIAM J. Optimal Control, 21, pp.246-255.

[47] Barmish, B. R., Petersen, I. R., and Feuer, A., 1983. "Linear Ultimate Boundedness Control for Uncertain Dynamical Systems", Automatica, 19, pp. 523-532.

[48] Yedavalli, R. K., and Devarakonda, N., "Ecological Sign Stability and its Use in Robust Control Design for Aerospace Applications," Proceedings of IEEE Conference on Control Applications, Sept. 2008.

[49] Yedavalli, R. K., and Sparks. A., "Satellite Formation Flying and Control Design Based on Hybrid Control System Stability Analysis," Proceedings of the American Control Conference, June 2000, pp. 2210.

[50] Nelson, R., Flight Stability and Automatic Control. McGraw Hill. Chap. 1998. 


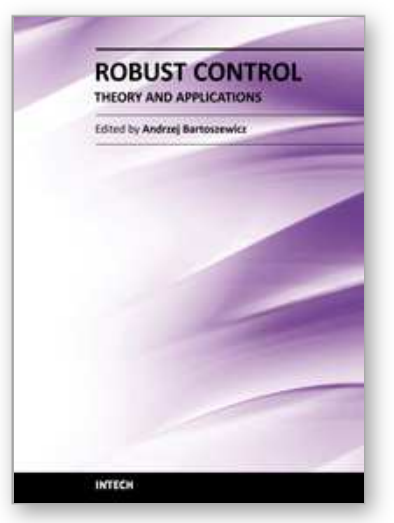

\author{
Robust Control, Theory and Applications \\ Edited by Prof. Andrzej Bartoszewicz
}

ISBN 978-953-307-229-6

Hard cover, 678 pages

Publisher InTech

Published online 11, April, 2011

Published in print edition April, 2011

The main objective of this monograph is to present a broad range of well worked out, recent theoretical and application studies in the field of robust control system analysis and design. The contributions presented here include but are not limited to robust PID, H-infinity, sliding mode, fault tolerant, fuzzy and QFT based control systems. They advance the current progress in the field, and motivate and encourage new ideas and solutions in the robust control area.

\title{
How to reference
}

In order to correctly reference this scholarly work, feel free to copy and paste the following:

Rama K. Yedavalli and Nagini Devarakonda (2011). Robust Stability and Control of Linear Interval Parameter Systems Using Quantitative (State Space) and Qualitative (Ecological) Perspectives, Robust Control, Theory and Applications, Prof. Andrzej Bartoszewicz (Ed.), ISBN: 978-953-307-229-6, InTech, Available from:

http://www.intechopen.com/books/robust-control-theory-and-applications/robust-stability-and-control-of-linearinterval-parameter-systems-using-quantitative-state-space-and

\section{INTECH}

open science | open minds

\section{InTech Europe}

University Campus STeP Ri

Slavka Krautzeka 83/A

51000 Rijeka, Croatia

Phone: +385 (51) 770447

Fax: +385 (51) 686166

www.intechopen.com

\section{InTech China}

Unit 405, Office Block, Hotel Equatorial Shanghai

No.65, Yan An Road (West), Shanghai, 200040, China

中国上海市延安西路65号上海国际贵都大饭店办公楼 405 单元

Phone: +86-21-62489820

Fax: $+86-21-62489821$ 
(C) 2011 The Author(s). Licensee IntechOpen. This chapter is distributed under the terms of the Creative Commons Attribution-NonCommercialShareAlike-3.0 License, which permits use, distribution and reproduction for non-commercial purposes, provided the original is properly cited and derivative works building on this content are distributed under the same license. 